\title{
CORBA-Based Open Platform for Processes Monitoring. An Application to a Complex Electromechanical Process
}

\author{
Karina Cantillo ${ }^{1}$, Rodolfo E. Haber ${ }^{1,2}$, Jose E. Jiménez ${ }^{1}$, Ángel Alique ${ }^{1}$, and \\ Ramón Galán ${ }^{3}$ \\ ${ }^{1}$ Instituto de Automática Industrial - CSIC, Campo Real km 0.200, \\ Arganda del Rey, Madrid 28500 \\ \{cantillo, rhaber, jejc, a.alique\}@iai.csic.es \\ ${ }^{2}$ Escuela Politécnica Superior. Ciudad Universitaria de Cantoblanco \\ Ctra. de Colmenar Viejo, km. 15. 28049 - Spain \\ Rodolfo.Haber@ii.uam.es \\ ${ }^{3}$ E.T.S. de Ingenieros Industriales, Universidad Politécnica de Madrid, \\ c/ José Gutiérrez Abascal №2, Madrid 28006. \\ rgalan@etsii.upm.es
}

\begin{abstract}
The goal of this work is to develop an open software platform called SYNERGY, supported by portable, low cost and worldwide-accepted technologies (i.e., Real Time CORBA), focused on networked control systems. Preliminary results of SYNERGY corroborate the viability for networked control, supervision and monitoring of complex electromechanical processes like high speed machining (HSM), on the basis of current communications and computation technologies upon open architectures.
\end{abstract}

\section{Introduction}

During the last decade, successful applications of distributed real time systems have grown considerably due mainly to the availability of new standards and open architectures based on distributed objects (e.g., middleware Common Object Request Broker Architecture CORBA). Indeed, the combination of these technologies with the current control and supervision techniques based on classical and Artificial Intelligence paradigms are the foundation for the development of the new generation of networked control systems (NCS) [1,2,3]. In order to deal with communication constraints in NCS, has increased the use of CORBA, which is structured in layers, ORB core, services and the application layer, easing the development of distributed applications [4].

This paper is organized as follows. The main characteristics of TAO (CORBA implementation) are shown in section 2. A brief description of HSM process is presented in section 3. The design and implementation of SYNERGY software platform and results concerning networked real-time monitoring of HSM process are presented in Section 4. Finally, some conclusions and remarks are provided. 


\section{Background}

TAO, The ACE (Adaptive Communication Environment) ORB, unlike the most of CORBA implementations in market (MT-Orbix, CORBAplus, Visiobroker, miniCOOL, Orbacus), provides a predictable behaviour. TAO real time ORB core shares a minimum part of ORB resources, reducing substantially the synchronization costs and the priority inversion between the process threads. These characteristics are responsible of a better performance of CORBA applications. Besides, TAO implement the specification Real-Time CORBA (RT CORBA), to support real-time distributed requirements, defining mechanisms and policies to control processor, communication and memory resources $[5,6,7]$.

\section{High Speed Machining Process}

In High speed machining (HSM), cutting force is considered to be the variable that best describes the cutting process. This can be used to evaluate the quality and geometric profile of the cutting surface, the tool wear and the tool breakage [8].

Relevant variables involved in HSM process are: cutting tool position $\left(x_{\mathrm{p}}, y_{\mathrm{p}}, z_{\mathrm{p}}\right)$ $\mathrm{mm}]$, spindle speed $(s)[\mathrm{rpm}]$, feed speed $(f)[\mathrm{mm} / \mathrm{min}]$, cutting power $\left(P_{c}\right)[\mathrm{kW}]$, cutting force $(F)[N]$, radial cutting depth $(a)[\mathrm{mm}]$ and cutting-tool diameter $(d)[\mathrm{mm}]$.

The laboratory at the CSIC is equipped with a KONDIA HS-1000 HSM centre and Siemens SINUMERIK840D open computerized numerical control (CNC). The communication between the CNC and the applications is done by a multiport interface (MPI). External signals of the sensors and acquisition cards are acquired and processed using a LABVIEW-based program called SignalAcquisition. The application NCDDE Server, supplied by Siemens [9], allows to access real time data in a machining centre.

\section{Results}

SYNERGY software platform consists in two main parts. A server application called Monitoring-Server that comprises data acquisition, identification and communication modules, and a client application called Remote-Monitoring, which includes communication and control modules. This work is focused on the data acquisition and the communication modules of the server and client application.

The communication modules were developed based in TAO (i.e., ACE5.3 TAO1.3 version). The TAO services and policies of RT CORBA used are: naming service, $R T$ ORB, RT POA, RT Current, Priority Mappings, Server_Declared Priority Model, Server Protocol Policy, Explicit Binding, Private Connections, POA Threadpools. The communication interface defined is depicted below.

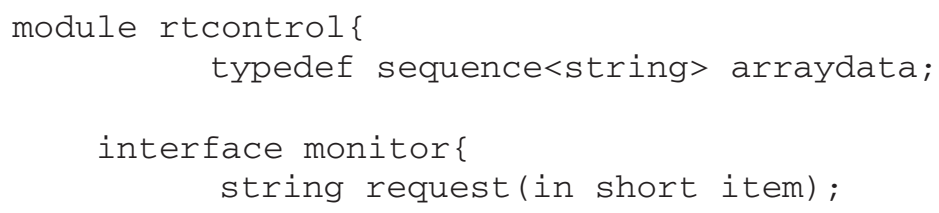




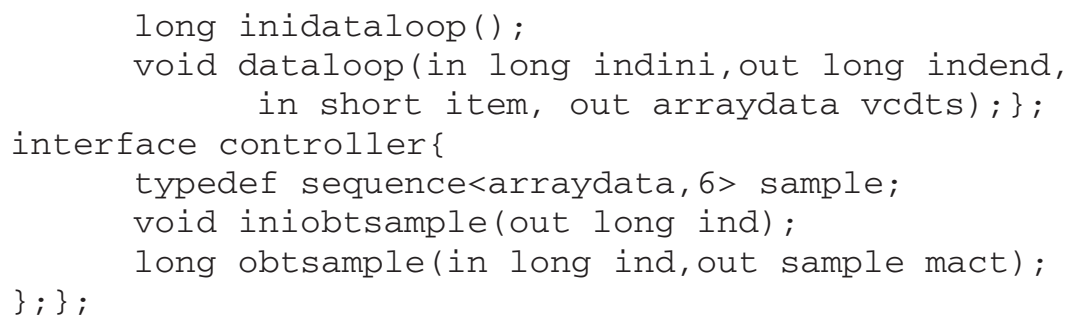

The acquisition module of Monitoring-Server application enables communications with the NCDDE Server and SignalAcquisition applications (Figure 1). Data are stored in a temporal matrix. Remote CORBA Object accesses the data matrix and recover data.

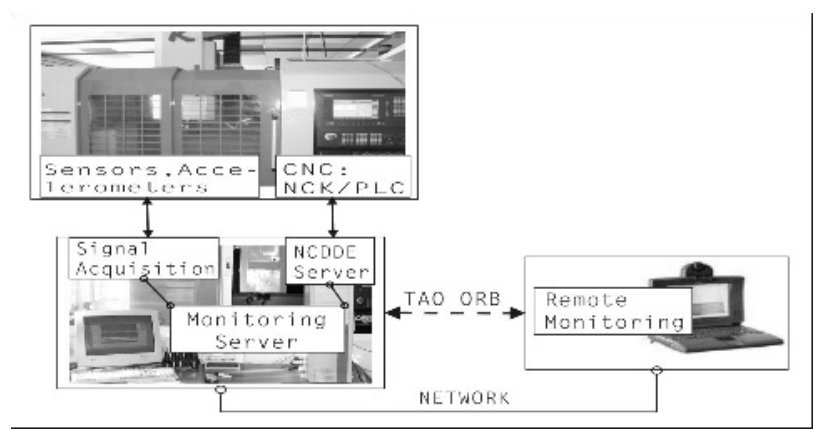

Fig. 1. Platform for networked control, supervision and monitoring of high speed machining

The developed platform was evaluated under real HSM operations. The condition of the cutting tool was also considered. The experiments were conducted considering high and low traffic in network for assessing the effect of the network congestion in the application performance. For the sake of space, only two cases are shown in Figure 2. For a case study with high network traffic the mean delay was $10.89 \mathrm{e}-3$ seconds and the variance of $6.897 \mathrm{e}-5 \mathrm{sec}$. In presence of low network traffic the mean delay was $8.8826 \mathrm{e}-3 \mathrm{sec}$. and the variance was $2.312 \mathrm{e}-4 \mathrm{sec}$.
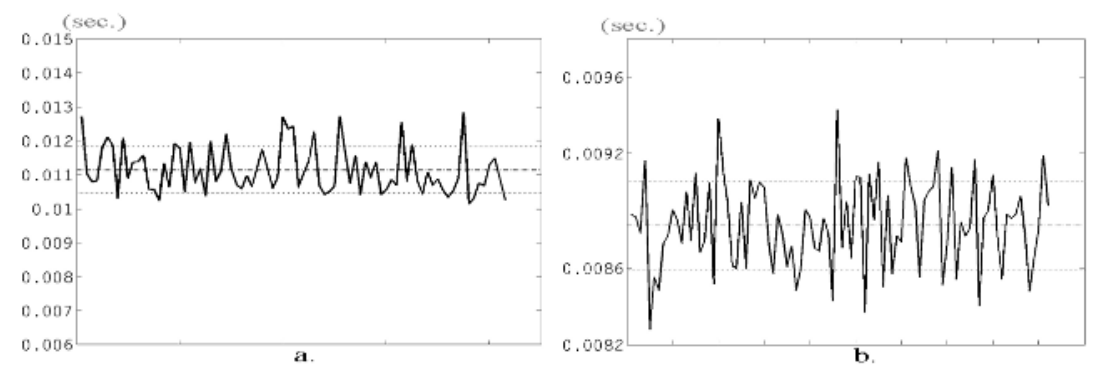

Fig. 2. Figure 2 a show the behaviour of the communication delay (measure in seconds) for high traffic and $2 b$ for low traffic 


\section{Conclusions}

An open architecture for measuring variables in a HSM process has been developed. Additionally, SYNERGY software platform has been designed for networked control, supervision and monitoring for HSM with the following characteristics:

- Portability, low cost design and easy implementation of the developed software, including the easiness to incorporate new technologies and control methodologies.

- Superior performance of real time TAO ORB for real time applications, providing a deterministic and predictable behaviour.

\section{References}

1. Hristu, D., Morgansen, K.: Limited Communication Control. Systems \& Control Letters, Elsevier Science B.V. 37 (1999) 193-205

2. Wong, W.S., Brockett, R.W.: Systems with Finite Communication Bandwidth Constraints II. Stabilization with Limited Information Feedback. IEEE Transactions on Automatic Control, Vol. 44, 5 (1999) 1049-1053

3. Haber R.E., Alique J.R.: Nonlinear internal model control using neural networks: Applications to machining processes, Neural Computing and Applications, Springer-Verlag London Limited, 2004 (DOI 10.1007/s00521-003-0394-8).

4. Sanz, R.: CORBA for Control Systems. IFAC Algorithms and Architectures for Real-Time Control, AARTC'2000. Palma de Mallorca, Spain (2000)

5. Schmidt, D.C., Levine, D.L., Mungee, S.: The Design of the TAO Real Time Object Request Broker. Computer Communications, Vol. 21, 4 (1998) 294-324

6. TAO Developer's Guide, Building a Standard in Performance. Object Computing, Inc. TAO version 1.2 a, Vol. 1, 2. St. Louis (2002)

7. Schmidt, D.C., Mungee, S., Gaitan, S.F, Gokhale, A.: Software Architectures for Reducing Priority Inversion and Non-determinism in Real-time Object Request Brokers. Journal of Real-time Systems, Special issue on Real-time Computing in the Age of the Web and the Internet Vol. 21, 1-2, (2001) 77-125

8. Haber R.E., Alique A., Alique J.R., Haber-Haber R., Ros S., Current trend and future developments of new control systems based on fuzzy logic and its application to high speed machining, Revista Metalurgia Madrid Vol. 38 (2002) 124-133

9. SINUMERIK 840D/810D/FM-NC. User Manual. Edition 09 2000, Siemens 